\title{
Influence of Early Life Stress on Intra- and Extra-Amygdaloid Causal Connectivity
}

\author{
Merida M Grant, ${ }^{*, 1}$ Kimberly Wood ${ }^{2}$, Karthik Sreenivasan ${ }^{3}$, Muriah Wheelock $^{2}$, David White', \\ Jasmyne Thomas ${ }^{4}$, David C Knight ${ }^{2}$ and Gopikrishna Deshpande ${ }^{4,5}$ \\ 'Department of Psychiatry and Behavioral Neurobiology, University of Alabama-Birmingham, Birmingham, AL, USA; '2Department of Psychology, \\ University of Alabama-Birmingham, Birmingham, AL, USA; ${ }^{3}$ Department of Electrical and Computer Engineering, AU MRI Research Center, Auburn \\ University, Auburn, AL, USA; ${ }^{4}$ Department of Neurobiology, University of Alabama-Birminghan, Birmingham, AL, USA; ${ }^{5}$ Department of Psychology, \\ Auburn University, Auburn, AL, USA
}

Animal models of early life stress (ELS) are characterized by augmented amygdala response to threat and altered amygdala-dependent behaviors. These models indicate the amygdala is a heterogeneous structure with well-differentiated subnuclei. The most well characterized of these being basolateral (BLA) and central nucleus (CeA). Parallel human imaging findings relative to ELS also reveal enhanced amygdala reactivity and disrupted connectivity but the influence of ELS on amygdala subregion connectivity and modulation of emotion is unclear. Here we employed cytoarchitectonic probability maps of amygdala subregions and Granger causality methods to evaluate task-based intra-amygdaloid and extra-amygdaloid connectivity with the network underlying implicit regulation of emotion in response to unconditioned auditory threat in healthy controls with ELS $(N=20)$ and without a history of ELS $(N=14)$. Groups were determined by response to the Childhood Trauma Questionnaire and threat response determined by unpleasantness ratings. Non-ELS demonstrated narrowly defined BLA-driven intra-amygdaloid paths and concise orbitofrontal cortex (OFC)-CeA-driven extra-amygdaloid connectivity. In contrast, ELS was associated with extensive and robust CeA-facilitated intra- and extra-amygdaloid paths. Non-ELS findings paralleled the known anatomical organization and functional relationships for both intra- and extra-amygdaloid connectivity, while ELS demonstrated atypical intra- and extra-amygdaloid CeA-dominant paths with compensatory modulation of emotion. Specifically, negative causal paths from OFC/BA32 to BLA predicted decreased threat response among non-ELS, while a unique within-amygdala path predicted modulation of threat among ELS. These findings are consistent with compensatory mechanisms of emotion regulation following ELS among resilient persons originating both within the amygdala complex as well as subsequent extra-amygdaloid communication. Neuropsychopharmacology (2015) 40, I782-1793; doi:I0.1038/npp.20I5.28; published online 25 February 2015

\section{INTRODUCTION}

Early life stress (ELS), specifically traumatic events that occur prior to age 18, represents a well-established risk factor for the adult onset of mood and anxiety disorders (Green et al, 2010). A shared feature of the neurophysiology of these illnesses is enhanced amygdala reactivity in response to aversive stimuli (Price and Drevets, 2010; Shin and Liberzon, 2010). Disruption of emotion regulation has been posited to underlie this augmented response and has largely been characterized in terms of illness-specific mechanisms such as increased sadness relative to depression or enhanced response to threat in anxiety disorders (Shin et al, 2005; Price and Drevets, 2010). Yet, recent findings relative to major depressive disorder (Grant et al, 2011) as well as resilience

*Correspondence: Dr MM Grant, Department of Psychiatry and Behavioral Neurobiology, University of Alabama-Birmingham, 1720 7th Avenue South, SC- 1006 Sparks Center, Birmingham, AL 37203, USA, Tel: +205975 5873, Fax: +205934 4655, E-mail: grantmm@uab.edu Received 2 September 2014; revised 13 January 2015; accepted 13 January 2015; accepted article preview online 29 January 2015
(Dannlowski et al, 2012), are more consistent with a generalized enhanced amygdala response to aversive stimuli following ELS, independent of diagnostic status.

Exposure to early adverse events and subsequent psychopathology is theorized to result principally from the influence of stress hormones on brain circuits related to the expression and inhibition of emotions such as fear (Rodrigues et al, 2009). However, to date, human imaging investigations of the interaction of stress and fear have primarily focused on either (1) acute mechanisms such as stress induction and exogenous corticosteroid modulation of response to threat in healthy volunteers (Merz et al, 2010; Merz et al, 2013) or (2) chronic stress mechanisms in psychiatric samples such as post-traumatic stress disorder (Williams et al, 2006). The effect ELS has on the circuit underlying response to threat both during early processing stages within the amygdala or the subsequent extra-amygdaloid subregion modulation of response in the absence of illness or medication effects remains unclear.

Proposed mechanisms generated from animal models include corticosteroid receptor factor and glucocorticoid receptor-mediated transformations in brain morphology, 
hyperexcitability of amygdala subnuclei (Vyas et al, 2003; Duvarci and Pare, 2007), and disinhibition via medial prefrontal cortex (mPFC) atrophy (Quirk et al, 2003; Radley et al, 2004). Parallel imaging findings in clinical and nonclinical samples have similarly demonstrated mPFC atrophy (Treadway et al, 2009), enhanced amygdala reactivity (Grant et al, 2011; Dannlowski et al, 2012), and altered restingstate and task-based connectivity (Philip et al, 2013; Grant et al, 2014). Thus, animal models of physiology and human imaging findings indicate that early onset and prolonged stress are associated with hypervigilance through both increased intrinsic excitability of amygdala, as well as disruption of inhibition.

The amygdala is a complex structure essential to the detection, valuation and expression of emotion (Zald, 2003) and modulation of its response is considered central to regulation of emotion. Specifically, effortful behaviors involving awareness and monitoring of goal-directed processes that function to influence the intensity, duration, and type of emotional experience are theorized to underlie explicit regulation, while implicit regulation is characterized by stimulus-driven, automatic processes without awareness (Gross and Thomspons, 2007). Although ELS has recently been demonstrated to negatively affect various cognitive and affective responses (Dannlowski et al, 2013), few studies have directly addressed the impact on modulation of emotion.

The amygdaloid complex is comprised of a number of heterogeneous nuclei and cortical regions with differential cytoarchitecture, physiology. and anatomical connections (cf., Freese and Amaral, 2009). Lateral nucleus is the primary site of sensory input via thalamic projections and input from cortical association areas of the brain. Projections from lateral nucleus extend to basal nucleus and together (BLA) underlie threat detection and consolidation of fear learning (LeDoux, 2007). BLA subsequently sends glutamatergic projections to central nucleus (CeA), which mediates defensive reactions via output to lateral hypothalamus (autonomic response), paraventricular nucleus (PVN; cortisol), and periaquaductal gray (freezing behavior). In contrast to BLA, the medial nucleus of CeA is thought to be primarily gamma-aminobutyric acid (GABA)-ergic (LeDoux, 2007).

Here we investigated intra-amygdaloid and extra-amygdaloid connectivity with the network underlying implicit regulation of emotion by employing cytoarchitectonic probabilistic maps of the amygdala. Based on animal models of intra-amygdaloid anatomy and physiology, we anticipated observing BLA-dominant, negatively predictive relationships with $\mathrm{CeA}$ and superficial nuclei (SF) in the non-ELS group (Davis, 2006; Roy et al, 2009) but not the ELS group. With regard to extra-amygdaloid connectivity, recent findings based on the uninstructed modulation of negative emotion demonstrate an inverse relationship between self-reported negative affect and ventral medial PFC (vmPFC) response, consistent with implicit regulation of emotion (Silvers et al, 2014). This finding is in contrast to response to instructed regulation of emotion which elicited dorsomedial and dorsolateral PFC response, consistent with explicit regulation of emotion. Here we evaluated the relationship between self-reported threat ratings in response to an unconditioned auditory threat stimulus and causal pathways in the brain, to determine whether activity in specific neural networks underlie implicit modulation of threat response. We anticipated findings similar to Silvers et al (2014), however we extended those findings to address neural networks (ie, we theorized that the causal path between vmPFC and amygdala would predict affective intensity) in addition to examining disparate amygdala subregion circuits. Differential connectivity for BLA and $\mathrm{CeA}$ has previously been observed in human imaging studies in healthy controls and those with anxiety disorders (ie, primarily cortical connectivity for BLA but subcortical connectivity for CeA; Etkin et al, 2009; Roy et al, 2009; Brown et al, 2014), however, these studies employed resting-state analysis. While resting-state studies are important to establish baseline activity within broad networks throughout whole brain, causal relationships cannot be discerned from simultaneous intrinsic activity. Thus, we employed Granger causality methods which rely on lagged time series data in one set of variables to predict future values of one or more other variables (Granger, 1969) and allow testing of previously established directional patterns of physiology from animal models of intra- and extra-amygdaloid communication.

This is the first study of ELS to our knowledge to investigate task-based causal connectivity in response to unconditioned threat, parsing amygdala into subregions, thus addressing the interaction of long term stress and fear on early processing of threat within amygdala in addition to subsequent extra-amygdaloid subregion modulation of emotion in the absence of illness or medication effects.

\section{MATERIALS AND METHODS}

\section{Participants}

Thirty-four healthy right handed controls participated in this study $(N=12$ males, $N=22$ females; age $=31 \pm 10.87$ years; range (18-59). All participants provided written informed consent in compliance with the University of Alabama at Birmingham Institutional Review Board. Participants for this analysis were primarily obtained from a larger ongoing study of stress and fear conditioning that employed conditioning paradigms (MMG). Data were pooled with a separate project investigating threat conditioning that used the same acquisition protocol performed on the same scanner at UAB in an independently acquired cohort (DCK). Both imaging projects were overseen by DCK based on an established paradigm described in detail previously (Woods et al, 2012). Subsequent analysis comparing the two groups on demographic and clinical measures found no significant differences and thus these data were pooled.

\section{Clinical Measures}

Structured clinical interview. All participants were evaluated using the Structured Clinical Interview for DSM-IV (SCID; (First et al, 2002). Clinical evaluations were performed by master's level and doctoral level clinicians in the department of psychiatry. Supervision and review was provided by MMG. Participants were between 19 and 55 years of age with no significant history of neurological disease or lifetime history of brain injury. Participants were required to have a score of six or less on the HDRS-17. All participants who met criteria were scheduled for a scan session. 


\section{Other Measures}

Childhood trauma questionnaire-short form. Groups were determined based on the childhood trauma score (see

Table I Demographic Data for Healthy Controls With and Without Early Life Trauma Stress

\begin{tabular}{|c|c|c|c|c|c|c|c|}
\hline \multirow[b]{2}{*}{ Variable } & \multirow{2}{*}{$\begin{array}{c}N \\
14\end{array}$} & \multicolumn{2}{|c|}{ Non-ELS } & \multirow{2}{*}{$\begin{array}{l}N \\
20\end{array}$} & \multicolumn{2}{|c|}{ ELS } & \multirow[b]{2}{*}{$p$-value } \\
\hline & & Mean & SD & & Mean & SD & \\
\hline $\operatorname{Sex}(F)$ & 10 & & & 12 & & & 0.72 \\
\hline Age & & 31.2 & 11.9 & & 30.8 & 10.5 & 0.90 \\
\hline Education & & 16.9 & 2.3 & & 16.4 & 2.0 & 0.58 \\
\hline Shipley & & 105.5 & 10.2 & & 105.9 & 10.2 & 0.92 \\
\hline HRSD-I7 & & 1.6 & 1.3 & & 2.1 & 0.8 & 0.27 \\
\hline $\mathrm{BDI}$ & & 0.8 & 1.0 & & 1.0 & 1.2 & 0.65 \\
\hline BAl & & I.I & 2.0 & & 0.7 & 0.9 & 0.58 \\
\hline CTQ Physical Abuse & & 5.9 & 0.9 & & 8.8 & 2.9 & 0.001 \\
\hline CTQ Sexual Abuse & & 5.0 & 0.0 & & 7.3 & 4.3 & 0.02 \\
\hline CTQ Emotional Abuse & & 6.3 & 1.9 & & 9.9 & 6.2 & 0.02 \\
\hline CTQ Emotional Neglect & & 6.6 & 1.9 & & 9.5 & 4.5 & 0.01 \\
\hline CTQ Physical Neglect & & 5.3 & 0.6 & & 6.7 & 2.3 & 0.02 \\
\hline PSS & & 28.8 & 6.0 & & 31.8 & 6.3 & 0.23 \\
\hline RSRI & & 2.0 & 0.36 & & 1.9 & 0.36 & 0.38 \\
\hline RLE & & 2.4 & 0.30 & & 2.14 & 0.36 & 0.11 \\
\hline
\end{tabular}

Abbreviations: BAI, Beck Anxiety Inventory; BDI, Beck Depression Inventory; CTQ, Childhood Trauma Questionnaire; HRSD-17, Hamilton Rating Scale for Depression- 17; PSS- I4, Perceived Stress Scale- |4; RLE, Recent Life Events; RSRI, Retrospective Self Report of Inhibition; Shipley, Shipley Institute for Living Scale. STAI data are based on a smaller sample from the pooled data set.
Table 1). All participants completed a well-validated measure of child abuse and neglect before the age of 18, the Childhood Trauma Questionnaire-Short Form (CTQ-SF; (Bernstein and Fink, 1998)). The measure includes 25 clinical questions with 5 factors that comprise physical, emotional and sexual abuse, and emotional and physical neglect. Participants rated statements about childhood experiences on a 5-point scale $(1=$ 'never true' to $5=$ 'very often true'. Scores were based on the mean value of the five individual items for each scale. The CTQ-SF has demonstrated high internal reliability, with (Cronbach's alphas ranging from 0.74 to 0.95 ) and good test-retest reliability at three months $(\mathrm{r}=0.80)$. The CTQ has also demonstrated good convergent validity with both a clinician-rated interview of childhood abuse and therapists' ratings of abuse (Fink et al, 1995). Because sexual abuse (CTQ-SA; Cronbach's alpha $=0.93-0.95$ ) and physical abuse (CTQ-PA alpha $=0.81-0.86)$ demonstrate greater reliability, we primarily focus on these scales in the current study. Additional clinical measures of stress and anxiety were also assessed (for more details, see Supplementary Methods).

\section{Imaging Paradigm}

As part of a larger ongoing study of Pavlovian conditioning in individuals with a history of ELS, participants completed conditioning paradigms that included both cued and uncued threat stimuli. Additional details on this methodology have been published previously (Wood et al, 2012).

Stimuli (conditioned and unconditioned). Two tones (700 and $1300 \mathrm{~Hz} ; 10 \mathrm{~s}$ duration; $20 \mathrm{~s} \mathrm{ITI)} \mathrm{served} \mathrm{as} \mathrm{the} \mathrm{condi-}$ tioned stimuli and a loud $(100 \mathrm{~dB})$ white-noise served as the unconditioned stimulus (duration: $0.5-6.0 \mathrm{~s}$ in 0.5 -s increments). The UCS co-terminated with one tone (CS+), while a

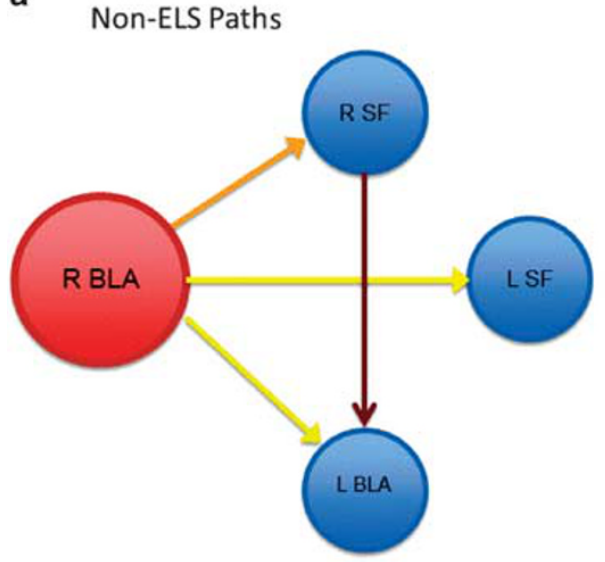

b

\section{ELS Paths}

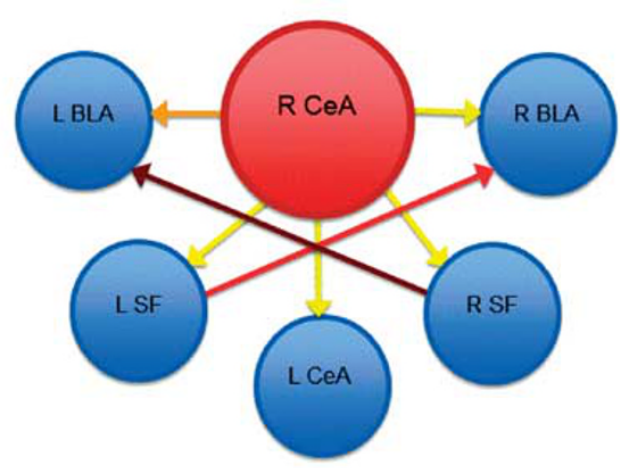

$6.12 \times 10^{-6}$

p-value

Figure I Intra-Amygdala Granger Causality Paths. Robust causal paths were observed for (a) Non-ELS participants. Right BLA-dominant causal paths were primarily observed, which predicted robust activity in left BLA, and left SF, with less robust relationships with right SF. An additional path from right SF to left BLA was also observed. This model is consistent with known anatomical and functional organization of the amygdala observed in animal models of threat. In contrast (b) ELS participants demonstrated primarily right CeA dominant paths with robust relationships with left CeA, bilateral SF and bilateral BLA. Additional relationships between left SF and right BLA and right SF and left BLA were also observed. This pattern was not consistent with known anatomical and functional organization of the amygdala in response to threat. p-values are FDR-corrected. Abbreviations: BLA, basolateral; CeA, central nucleus; ELS, early life stress; FDR, false discovery rate; SF, superficial nuclei. 
Table 2 Intra-Amygdala Network Granger Causality

\begin{tabular}{lllll}
\hline ELS ROI & \multicolumn{3}{c}{ MNI } & Mean conn \\
\cline { 2 - 4 } & $\mathbf{X}$ & $\mathbf{Y}$ & $\mathbf{Z}$ & \\
& & & &
\end{tabular}

R CeA to

$\begin{array}{lrrrr}\text { L CeA } & -22 & -4 & -12 & 0.06 \\ \text { L BLA } & -22 & -3 & -14 & 0.07 \\ \text { R BLA } & 28 & -4 & -12 & 0.06 \\ \text { L SF } & -22 & 0 & -15 & 0.07 \\ \text { R SF } & 26 & 0 & -12 & 0.07\end{array}$

LSF to

$\begin{array}{rrrrrc} & \text { R BLA } & 26 & -3 & -17 & 0.06 \\ \text { R SF to } & \text { L BLA } & -22 & -3 & -14 & -0.05\end{array}$

Non-ELS

Mean conn

R BLA to

$\begin{array}{crrrr}\text { L BLA } & -27 & -1 & -20 & 0.08 \\ \text { LSF } & -22 & 0 & -15 & 0.09 \\ \text { R SF } & 26 & 0 & -12 & 0.08\end{array}$

R SF to

$$
\text { L BLA }
$$$$
-22
$$$$
-3
$$$$
-14
$$

0.06

Abbreviations: BLA, basolateral; CeA, central nucleus; ESF, superficial nuclei; L, left hemisphere; mean conn, mean connectivity coefficient; MNI, Montreal Neurological Institute; R, right hemisphere.

Note: Causal connectivity between amygdala subregions. Coordinates are in $\mathrm{MNI}$. Coordinates indicate location of local maxima. $p$-values $<0.05$, FDR corrected. the second tone was presented alone $(\mathrm{CS}-)$ during acquisition (two $960 \mathrm{~s}$ blocks). The acquisition phase also included presentations of the UCS alone to contrast the unconditioned response with the conditioned response. A total of $24 \mathrm{CS}+$, 24 CS -, and 24 UCS alone trials were presented during acquisition. Stimuli were counterbalanced and presented in a pseudorandomized order such that no more than two trials of the same stimulus were consecutively presented. The current analysis addressed only those trials that involved the UCS alone, as we were interested in intra- and extraamygdaloid connectivity in response to the UCS. (For more details, see Supplementary Methods).

Unpleasantness ratings. Participants completed in-scanner ratings of the unpleasantness of the UCS following each of two acquisition runs on a scale from 0 (not unpleasant) to 10 (unpleasant). Ratings were averaged across runs per group.

\section{Functional MRI}

Scans were acquired using a $3 \mathrm{~T}$ Siemens Allegra head only scanner at the University of Alabama at Birmingham. Highresolution structural images (MPRAGE) were acquired in the sagittal plane using a $\mathrm{T} 1$ weighted series $(\mathrm{TR}=2300 \mathrm{~ms}$, $\mathrm{TE}=3.9 \mathrm{~ms}$, flip angle $=12^{\circ}, \mathrm{FOV}=25.6 \mathrm{~cm}$, matrix $=256 \times$ 256 , slice thickness $=1 \mathrm{~mm}, 0.5 \mathrm{~mm}$ gap). Thirty-four whole brain slices were acquired in an interleaved pattern using a gradient-echo echoplanar pulse sequence in an oblique-axial orientation $\left(\mathrm{TR}=2000 \mathrm{~ms}, \mathrm{TE}=30 \mathrm{~ms}\right.$, flip angle $=70^{\circ}$, FOV $=24 \mathrm{~cm}$, matrix $=64 \times 64$, slice thickness $=4 \mathrm{~mm}$, no gap). Images were normalized to MNI space, saved with a spatial resolution of $1.5 \times 1.5 \times 1.5 \mathrm{~mm}^{3}$, and smoothed using a $4-\mathrm{mm}$ full-width at half maximum Gaussian kernel

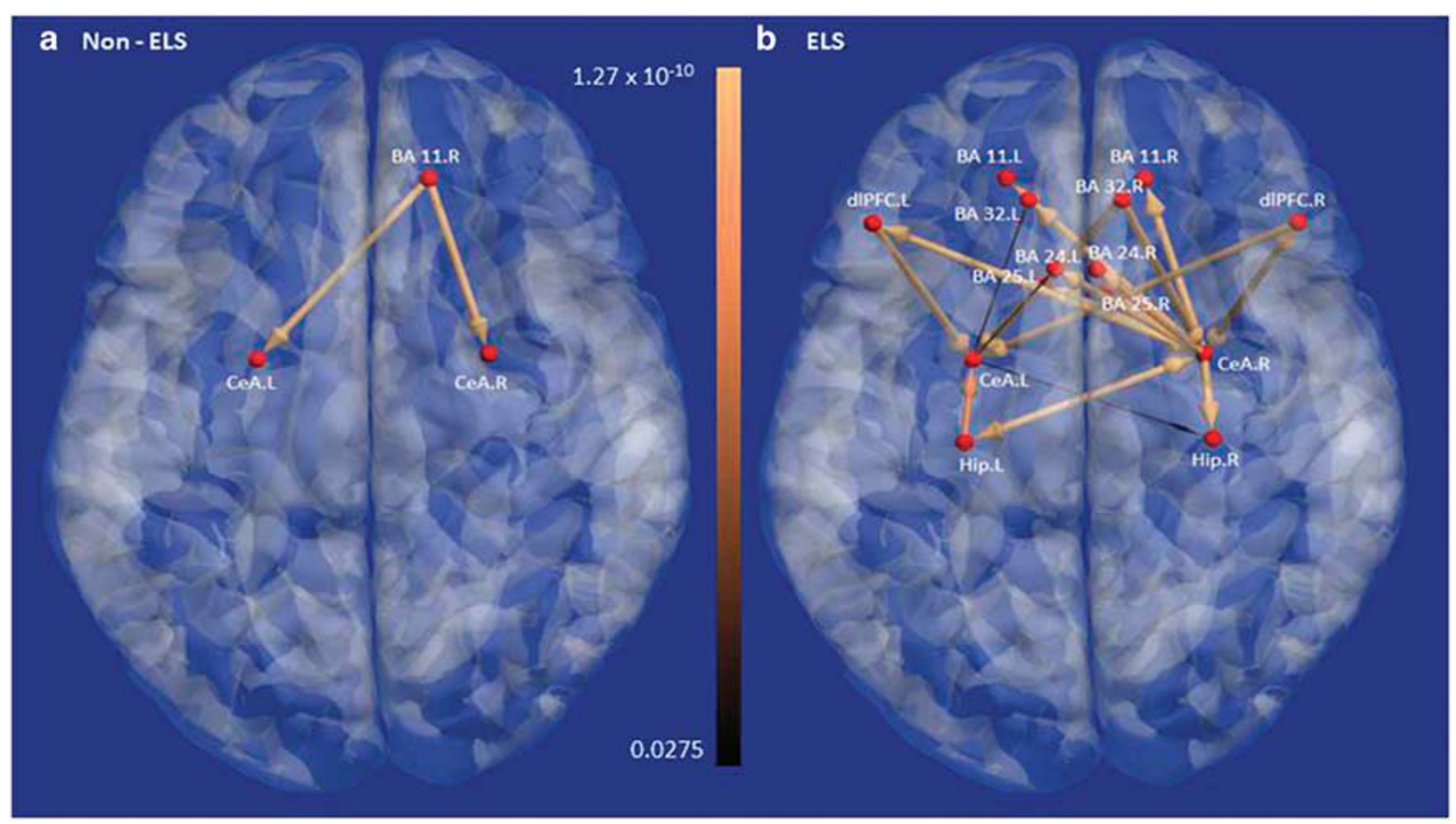

Figure 2 Extra-Amygdaloid Granger Causality Paths: CeA. (a) Non-ELS demonstrated a concise OFC-derived path that predicted bilateral CeA response. In contrast, the ELS group demonstrated a much more complex CeA-dominant pattern in which right CeA activity predicted activity in multiple cortical regions including bilateral DLPFC, BA I I, BA32, 25 and 24, and hippocampus. In addition right DLPFC predicted bilateral CeA, while left DLPFC predicted left CeA. Left hippocampus predicted bilateral CeA. p-values are FDR-corrected. Abbreviations: BA, Brodmann's Area; CeA, central nucleus; DLPFC, dorsal lateral prefrontal cortex; ELS, early life stress; FDR, false discovery rate; OFC, orbitofrontal cortex. 
Table 3 Extra-Amygdaloid Granger Causality Paths (CeA)

\begin{tabular}{lllll}
\hline ELS ROI & \multicolumn{3}{c}{ MNI } & Mean conn \\
\cline { 2 - 4 } & & & \\
& & $Y$ & $Y$ & $Z$
\end{tabular}

BA 24L to

$\begin{array}{lllll}\mathrm{LCeA} & -22 & -4 & -12 & 0.05\end{array}$

BA $32 L$ to

$\begin{array}{lllll}\mathrm{LCeA} & -21 & -9 & -11 & -0.05\end{array}$

$B A 32 R$ to

$\begin{array}{lrrrr}\text { LCeA } & -21 & -9 & -11 & 0.07 \\ \text { R CeA } & 22 & -4 & -11 & 0.09\end{array}$

$D L P F C L$ to

$\begin{array}{lrrrr}\text { LCeA } & -21 & -9 & -11 & 0.07 \\ \text { R CeA } & 22 & -4 & -12 & 0.06\end{array}$

DLPFC R to

$\begin{array}{lrrrr}\text { LCeA } & -22 & -4 & -12 & 0.07 \\ \text { R CeA } & 22 & -4 & -11 & 0.08\end{array}$

Hipp L to

$\begin{array}{lrrrr}\text { LCeA } & -22 & -4 & -11 & -0.06 \\ \text { R CeA } & 22 & -4 & -11 & -0.06\end{array}$

$L C e A$ to

$\begin{array}{lrrrr}\text { BA 24L } & -10 & 15 & 31 & 0.06 \\ \text { BA 32L } & 4 & 36 & 25 & 0.05 \\ \text { Hipp R } & -22 & -24 & -8 & 0.05\end{array}$

$R$ CeA to

Non- ELS

\begin{tabular}{lrrrr} 
BA IIL & -24 & 48 & -6 & 0.06 \\
BA IIR & 22 & 42 & -15 & 0.07 \\
BA 24L & -4 & 21 & 28 & 0.07 \\
BA 24R & 4 & 24 & 28 & 0.06 \\
BA 25L & -6 & 20 & -2 & 0.07 \\
BA 32L & -4 & 23 & 31 & 0.08 \\
DLPFC L & -54 & 8 & 39 & 0.07 \\
DLPFC R & 43 & 30 & 36 & 0.06 \\
Hipp L & -18 & -24 & -8 & 0.07 \\
Hipp R & 26 & -28 & -9 & 0.07 \\
& & & & Mean conn \\
L CeA & -21 & -9 & -11 & 0.09 \\
R CeA & 22 & -4 & -11 & 0.09 \\
\hline
\end{tabular}

Abbreviations: BA I I, Brodmann's Area II; CeA, central nucleus; DLPFC, dorsolateral prefrontal cortex; Hipp, hippocampus; L, left hemisphere; mean conn, mean connectivity coefficient; MNI, Montreal Neurological Institute; R, right hemisphere.

Note: Granger Causality Paths between $\mathrm{CeA}$ and implicit regulation of emotion network. $p$-values $<0.05$, FDR corrected. Coordinates are in MNI. Coordinates indicate location of local maxima. consistent with prior investigations employing similar methodology to reduce the likelihood of spatial localization error (Gamer et al, 2010). Regressors representing trial type (CS+, CS - , and UCS) were modeled with a canonical hemodynamic response function, although only UCS trials were included in the current analysis. Movement parameters from the realignment stage were included in the model as covariates of no interest. Second-level random effects analysis was employed for group comparisons based on trauma history (for more details, see Supplementary Methods).

\section{Probability Maps and Region of Interest (ROI) Analysis}

Six bilateral regions were selected based on the literature elaborating amygdala subregions and their role in translational models of fear conditioning (Amaral et al, 1992, LaBar and LeDoux, 2011). Seed regions were based on probability maps derived from the Anatomy Toolbox in SPM5 (Eickhoff et al, 2005). Time series data were extracted for all voxels with at least an $80 \%$ probability of belonging to one of the three subregions (lateral nucleus and BLA, CeA, or superficial including anterior amygdaloid area, amygdalopyriform transition area, amygdaloid-hippocampal area and ventral and posterior cortical nuclei (SF)) for right and left hemisphere (Amunts et al, 2005).

\section{Effective Connectivity Model}

In this study we utilized the method of Granger Causality (GC) to perform effective connectivity analysis. GC is based on the principle that the causal influence of one region $\mathrm{X}$ on another region $\mathrm{Y}$ can be obtained if past values of the time series from the region $\mathrm{X}$ help predict the present and future values of the time series from the region Y (Granger, 1969). This method is implemented using a multivariate auto regressive (MVAR) model (for more details, See Supplementary Methods).

\section{Granger Causality Analysis}

The mean time series from these ROI were extracted for all participants (Supplementary Figure S1). These average time series were temporally normalized and the latent neuronal state variables were obtained by hemodynamic deconvolution of the fMRI time series using the cubature Kalman filter (Havlicek et al, 2011). A boxcar function corresponding to the input stimulus (UCS) was used as the exogenous input to the deconvolution model along with normalized fMRI time series from previously identified activated ROIs. The hidden neuronal variables obtained after deconvolution were input into a dynamic MVAR model to obtain dynamic effective connectivity between every pair of ROIs for all the participants. Samples of task specific connectivities were obtained by populating the Granger causality values from all participants based on the UCS condition. The DGC connectivity metrics corresponding to the UCS alone condition were populated into separate samples for the two participant groups (ELS and non-ELS participants). The mean of these samples are denoted as the mean connectivity values in the tables. All $p$-values generated 
from these analyses were corrected for multiple comparisons using the false discovery rate.

\section{Correlation Between Unpleasantness Ratings and Granger Causality}

As a measure of implicit regulation of emotion, we performed correlation analysis between mean connectivity coefficients for intra-and extra-amygdaloid paths and individual threat ratings for the UCS. Participants were not asked explicitly to regulate their response to auditory threat but were merely asked to rate each block of stimuli.

\section{RESULTS}

Demographic and clinical characteristics of this sample are presented in Table 1. No significant between-group differences were observed for any of the demographic variables. Nor did the groups differ on clinical measures of mood or anxiety. The anticipated differences in trauma history were noted for each of the five CTQ subscales, with the most robust difference observed for physical abuse. The means for both groups, however, were consistent with nonclinical samples.

\section{GC and Intra-Amygdaloid Paths}

Non-ELS. Within the non-ELS group, effective connectivity analysis revealed a series of robust intra-amygdaloid paths primarily originating from right BLA in response to the UCS (Figure 1a; Table 2). The only path that did not originate from right BLA was observed from right SF to left BLA and was less robust than the BLA paths.
ELS. Within the ELS group much more extensive taskbased connectivity was observed for each of the three amygdala subregions evaluated. In contrast to the non-ELS group, an opposing pattern was observed with right CeA predictive paths, rather than BLA (Figure 1b; Table 2). The most robust of these paths were from CeA to bilateral SF and bilateral BLA, respectively. Two less robust paths were observed from left SF to right BLA and from right SF to left BLA. No significant paths originated from either hemisphere for BLA in this group. (For group comparisons, see Supplementary Results).

\section{GC and Extra-Amygdaloid Paths}

Non-ELS. Within the non-ELS group, a concise taskdriven predictive path from right OFC (BA11) to bilateral CeA (Figure 2a; Table 3) was observed in response to the UCS. A mutual predictive path in both directions was observed between right BLA and right OFC (Figure 3a; Table 4). In addition, causal paths originating in right BLA to bilateral BA 32, left dorsal lateral prefrontal cortex (DLPFC), and right hippocampus were also observed. Similar to the pattern demonstrated with CeA, the non-ELS group demonstrated concise causal connectivity between right OFC and bilateral SF (Figure 4a; Table 5). An additional path between left SF and right BA 24 was also observed.

ELS. In contrast, the ELS group demonstrated predictive paths primarily originating from bilateral CeA (primarily right hemisphere) to numerous lateral and medial PFC regions, as well as bilateral hippocampus (Figure 2b; Table 3). Positive causal paths between bilateral DLPFC and bilateral CeA were observed in addition to paths originating from bilateral BA 32,

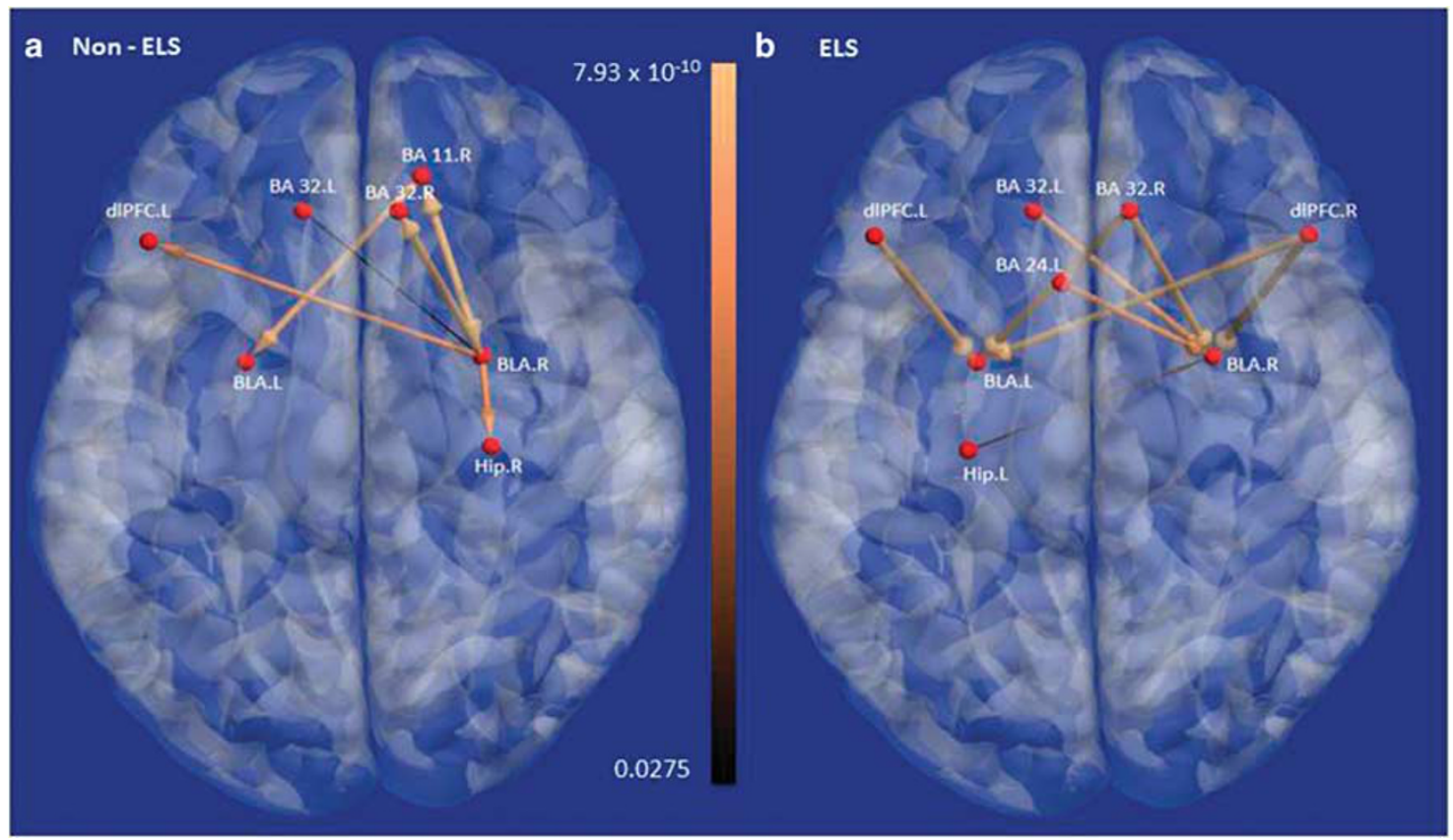

Figure 3 Extra-Amygdaloid Granger Causality Paths: BLA. (a) Non-ELS demonstrated BLA-dominant causal connectivity that predicted activity in bilateral BA 32, right BAII, right hippocampus, and left DLPFC. In addition BAI I predicted bilateral BLA. (b) Multiple cortical regions predicted bilateral BLA activity including right DLPFC, BA 32, left BA32 and BA24 which predicted right BLA. Right BA32, right DLPFC and left DLPFC predicted left BLA response. Left hippocampus also predicted right BLA response. p-values are FDR-corrected. Abbreviations: BA, Brodmann's Area; BLA, basolateral; DLPFC, dorsal lateral prefrontal cortex; ELS, early life stress; FDR, false discovery rate. 
Table 4 Extra-Amygdaloid Granger Causality Paths (BடA)

\begin{tabular}{|c|c|c|c|c|c|}
\hline \multirow[t]{2}{*}{ ELS } & \multirow[t]{2}{*}{ ROI } & \multicolumn{3}{|c|}{ MNI } & \multirow[t]{2}{*}{ Mean conn } \\
\hline & & $x$ & $\mathbf{Y}$ & $\mathbf{Z}$ & \\
\hline
\end{tabular}

BA $24 L$ to

$\begin{array}{lllll}\text { BLA R } & 28 & -4 & -12 & 0.06\end{array}$

BA $32 L$ to

$\begin{array}{lllll}\text { BLA R } & 26 & -3 & -17 & -0.05\end{array}$

BA $32 R$ to

$\begin{array}{lllll}\text { BLA L } & -22 & -3 & -14 & 0.08\end{array}$

$D L P F C L$ to

$\begin{array}{lllll}\text { BLA R } & 26 & -3 & -17 & 0.08\end{array}$

$\begin{array}{lllll}\text { BLA L } & -27 & -1 & -20 & 0.06\end{array}$

DLPFC R to

$\begin{array}{lllll}\text { BLA L } & -27 & -1 & -17 & 0.10\end{array}$

$\begin{array}{lllll}\text { BLA R } & 26 & -3 & -17 & 0.09\end{array}$

Hipp L to

\begin{tabular}{lcrccc} 
Non-ELS & BLA R & 28 & -4 & -12 & $\begin{array}{c}-0.05 \\
\text { Mean conn }\end{array}$ \\
\hline BA IIR to & & & & & \\
& BLA L & -27 & -1 & -20 & 0.07 \\
& BLA R & 26 & -3 & -17 & 0.08
\end{tabular}

$B L A R$ to

$\begin{array}{lrrrr}\text { BA IIR } & 24 & 5 I & -9 & 0.08 \\ \text { BA 32L } & -2 & 32 & 30 & 0.07 \\ \text { BA 32R } & 4 & 36 & 25 & 0.10 \\ \text { DLPFC L } & -40 & 3 & 28 & 0.08 \\ \text { Hipp R } & 16 & -31 & -5 & 0.08\end{array}$

Abbreviations: BA I I, Brodmann's Area II; DLPFC, dorsolateral prefrontal cortex; Hipp, hippocampus; L, left hemisphere; mean conn, mean connectivity coefficient; $M N I$, Montreal Neurological Institute; $R$, right hemisphere.

Note: Granger causality paths between basolateral nucleus (BLA) and implicit regulation of emotion network. Coordinates are in $\mathrm{MNI}$. Coordinates indicate location of local maxima. $p$-values $<0.05$, FDR corrected.

hippocampus, and left BA 24 to bilateral CeA. The ELS group was also characterized by more distributed causal paths between BLA and medial/lateral PFC, and left hippocampus to bilateral BLA (Figure 3b; Table 4). A robust inverse causal relationship was observed between left BA 32 and right BLA. Projections from bilateral DLPFC to BLA were also observed. This group also demonstrated more extensive causal connectivity patterns for SF, governed primarily by paths originating in either bilateral BA 32 or DLPFC (Figure 4b; Table 5). Additional paths originating in left BA 24 and left hippocampus to bilateral SF were also observed. Negative causal paths from right SF to BA 11, BA 25, and BA 32 were also elicited. (For group comparisons, see Supplementary Results).

\section{Correlation Between Unpleasantness Ratings and Granger Causality}

Significant positive correlations were observed between mean connectivity coefficients and threat ratings for extraamygdaloid paths in the non-ELS group (Figure 5) for right OFC to left BLA and left BA32 to left BLA. A negative correlation between the right hippocampus-right BLA path and perceived threat was also observed in the non-ELS group. In contrast, the intra-amygdaloid path from right BLA to left CeA and perceived threat demonstrated a negative correlation in the ELS group.

\section{DISCUSSION}

To our knowledge, the current study is the first to examine task-based Granger causality both within the amygdaloid complex as well as connectivity with the circuit implicated in implicit regulation of emotion based on trauma history in the absence of illness or treatment effects. Healthy volunteers with no history of ELS demonstrated a narrowly defined, task-specific BLA-driven intra-amygdaloid connectivity pattern consistent with electrophysiological studies in animal models of threat (LeDoux, 2007). In contrast, a history of ELS was associated with extensive and robust CeA-facilitated paths both within the amygdaloid complex and in relation to the circuit underlying implicit regulation of emotion. Given the relevance of CeA for fear expression, stress-related glucocorticoid output, and autonomic arousal, this pattern has the potential to underlie significant risk for psychiatric illness.

\section{Intra-Amygdaloid Granger Causality}

Communication between subregions within the amygdaloid complex is central to the detection, evaluation, and expression of emotion. Within the non-ELS group, network paths originating in right BLA robustly predicted subsequent activity within the amygdala that parallel the known anatomical organization and functional relationships derived from animal models in which association areas and thalamic nuclei provide somatosensory input to BLA. This input is subsequently conveyed to $\mathrm{CeA}$ and SF, facilitating expression of threat response and autonomic output (LeDoux, 2007; LaBar and LeDoux, 2011).

In contrast, the ELS group was characterized by a pattern of intra-amygdaloid network GC connectivity that did not comport with customary anatomical organization and functional models of auditory conditioning. BLA activity did not predict subsequent $\mathrm{CeA}$ response as would be expected. Instead, activity in bilateral CeA predicted ensuing response in left BLA and bilateral SF. This finding is contrary to known anatomical organization and functional relationships identified in prior animal and imaging studies of threat response in the amygdala (LeDoux, 2007; Roy et al, 2009). We speculate that since central medial neurons are known to be primarily GABAergic in nature, the inverse causal path from CeA to BLA may reflect an inhibitory feedback mechanism that dampens overall amygdala activity associated with behavioral hypervigilance and fear, thus short-circuiting potential pathophysiological output and maintaining resilience. 


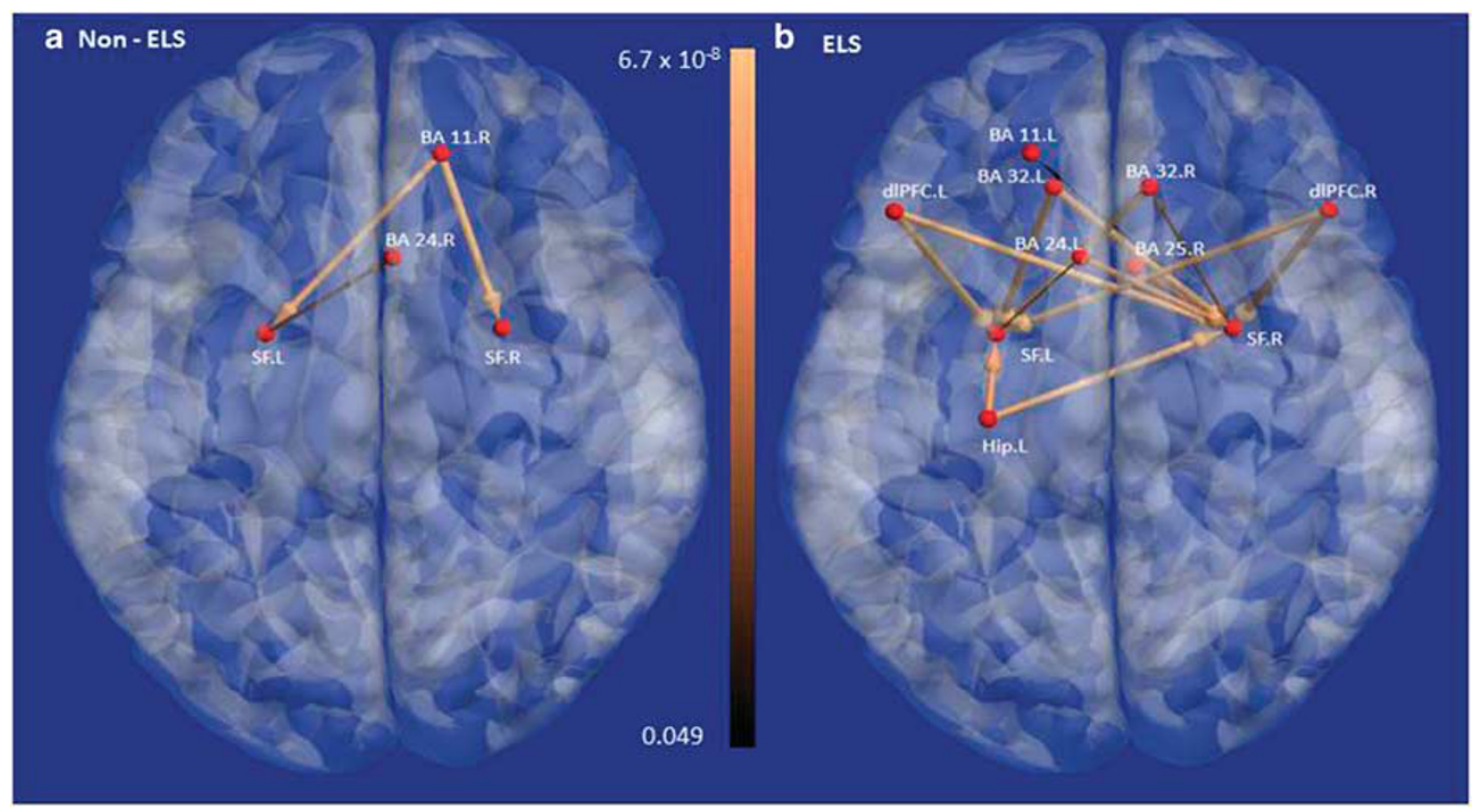

Figure 4 Extra-Amygdaloid Granger Causality Paths: SF. (a) Non-ELS demonstrated a concise OFC-dominant causal connectivity that predicted bilateral SF. In addition, left SF predicted right BA24 activity. (b) Again multiple cortical regions predicted right SF response including right DLPFC and BA32 and left DLPFC, BA32, BA24 and hippocampus. Left SF was predicted by left DLPFC, BA32, BA24 and hippocampus, as well as right DLPFC, BA32. p-values are FDRcorrected. Abbreviations: BA, Brodmann's Area; DLPFC, dorsal lateral prefrontal cortex; ELS, early life stress; FDR, false discovery rate; OFC, orbitofrontal cortex; SF, superficial nuclei.

\section{Extra-Amygdaloid Granger Causality}

Communication between the amygdala and the cortical network underlying implicit regulation of emotion within the non-ELS group demonstrated a concise pattern in which activity in right OFC (mPFC) predicted response in bilateral $\mathrm{CeA}$ and SF. Evidence from animal models and probabilistic tractography in humans demonstrate both an anatomical and functional relationship between OFC and amygdala in general and specifically with superficial nuclei (CeA/SF) but not BLA (Ghashghaei et al, 2007; Bach et al, 2011). These reciprocal projections between OFC and amygdala have been posited to underlie mutual inhibition.

In contrast, extra-amygdaloid connectivity within the ELS group was much more complex both with regard to (1) the primary direction of pathways from CeA to cortex, instead of cortex to amygdala, and (2) the total number of robust paths. Notably, the majority of these paths did not originate in either dorsal or vmPFC, which would be consistent with cortical inhibition, nor were they bidirectional, which would reflect mutual inhibition. Instead, the pattern was bottom up, which seems more consistent with a non-clinical level of cortical hyperarousal motivated by $\mathrm{CeA}$. In contrast to the simple causal path between right OFC and bilateral CeA among the non-ELS group, the few cortical paths observed for ELS originated primarily from bilateral DLPFC and right BA 32 to CeA in what appears to be a compensatory mechanism of inhibition.

Investigations of regulation of emotion in healthy volunteers have reliably demonstrated a role for DLPFC in the modulation of amygdala response (Meyer-Lindenberg et al, 2005; Banks et al, 2007; Stein et al, 2007; Wheelock et al, 2014). Despite sparse, unidirectional, ascending projections from amygdala to DLPFC observed in trace studies (Ghashghaei and Barbas, 2002), functional connectivity between these structures is theorized to underlie cortical inhibition of amygdala output, though indirectly.

Moreover, Ghashghaei et al (2007) in their review of non-human primate anatomy and neurochemistry identified prefrontal regions that direct output to amygdala $v s$ those that primarily receive input. Brain regions identified primarily as 'senders' include dorsal and ventral BA 24, BA 32, and BA 11. Our findings were consistent with these anatomical relationships as well as with more recent findings differentiating deep and superficial nuclei (Bach et al, 2011).

\section{Implicit Regulation of Emotion}

Despite the observation of robust medial and lateral cortical connectivity with amygdala subregions in response to the UCS, modulation of the threat response in the non-ELS group corresponded only with paths between vmPFC and amygdala. These findings are consistent with prior theories of the modulation of emotion that ascribe automatic processing to mPFC (Phillips et al, 2008). Moreover, no significant relationships were observed between intra-amygdaloid paths and threat ratings. This suggests that direct feedforward communication from BLA (threat detection and fear memory) to CeA (fear expression) was not central to perceived experience of threat in individuals with no history of ELS. Notably, negative predictive causal paths were consistently observed from mPFC to BLA but not directly with CeA. Thus, among non-ELS, inhibition of threat detection and/or consolidation of fear memory, may underlie modulation of emotion in the absence of direct inhibition of fear expression. 
Table 5 Extra-Amygdaloid Granger Causality Paths (SF)

\begin{tabular}{|c|c|c|c|c|c|}
\hline \multirow[t]{2}{*}{ ELS } & \multirow[t]{2}{*}{ ROI } & \multicolumn{3}{|c|}{ MNI } & \multirow[t]{2}{*}{ Mean conn } \\
\hline & & $x$ & $\mathbf{Y}$ & $\mathbf{Z}$ & \\
\hline
\end{tabular}

BA 24L

$\begin{array}{rrrrr}\text { SF L } & -22 & 0 & -15 & 0.05 \\ \text { SF R } & 26 & 0 & -12 & 0.06\end{array}$

BA $32 L$

$\begin{array}{rrrrr}\text { SF L } & -14 & -7 & -17 & -0.06 \\ \text { SF R } & 26 & 0 & -12 & -0.06\end{array}$

BA $32 R$

$\begin{array}{lllll}\text { SF L } & & & & 0.07 \\ \text { SF R } & 26 & 0 & -12 & 0.07\end{array}$

DLPFC L

$\begin{array}{lrrrr}\text { SF L } & -22 & 0 & -15 & 0.07 \\ \text { SFR } & 26 & 0 & -12 & 0.08\end{array}$

DLPFC R

$\begin{array}{lrrrr}\text { SF L } & -22 & 0 & -15 & 0.07 \\ \text { SFR } & 26 & 0 & -12 & 0.07\end{array}$

Hipp L

$\begin{array}{rrrrr}\text { SF L } & -14 & -7 & -17 & -0.06 \\ \text { SFR } & 26 & 0 & -12 & -0.06\end{array}$

SF R

$\begin{array}{lrrrr}\text { BA IIL } & -30 & 50 & -11 & -0.05 \\ \text { BA 25R } & 4 & 3 & -5 & -0.05 \\ \text { BA 32R } & 4 & 36 & 25 & -0.05\end{array}$

Non-ELS

BA $32 R$

Mean conn

$B A \mid / R$

$\begin{array}{lrrrr}\text { SF L } & -14 & -7 & -17 & 0.07 \\ \text { SF R } & 26 & 0 & -12 & 0.10\end{array}$

SF L

BA 24R

$4 \quad 24$

28

0.06

Abbreviations: BA I I, Brodmann's Area I I; DLPFC, dorsolateral prefrontal cortex; Hipp, hippocampus; L, left hemisphere; mean conn, mean connectivity coefficient; MNI, Montreal Neurological Institute; R, right hemisphere; SF,

superficial nuclei.

Note: Granger causality paths between SF and implicit regulation of emotion network. Coordinates are in MNI. Coordinates indicate location of local maxima. $p$-values $<0.05$, FDR corrected.

No significant relationships were observed among the ELS group for extra-amygdaloid connectivity and modulation of emotion. These finding was consistent with prior observations of attenuated emotion regulation among traumaexposed adolescents (Marusak et al, in press) and depressed adults with and without a history of ELS (Grant et al, 2014). Instead a negative relationship between the intra-amygdaloid
BLA-CeA path and threat was observed. Thus it appears that modulation of perceived threat following ELS is not driven by either medial or lateral cortical inhibition of amygdala but instead by within-amygdala inhibition of fear expression. These findings suggest that a history of ELS in healthy controls is associated with compensatory modulation of emotion within the amygdala itself that is not dependent on lateral PFC mechanisms that involve working memory or self-monitoring (Kalisch, 2009), nor vmPFC mechanisms that rely on learning, memory, or updating of affective value in response to feedback (Schiller et al, 2008). Instead, these individuals appear to rely on a unique compensatory mechanism within the amygdala, such that extra-amygdaloid alterations in brain morphology and physiology that are linked to long-term stress do not result in persistent pathological disruption of emotion regulation.

\section{Summary}

We observed disparate causal pathways for both intra- and extra-amygdaloid connectivity based on trauma history. In particular non-ELS was consistent with the well-defined intraamygdaloid anatomical and physiological pattern observed in animal models of threat, as well as a concise model of OFCmediated connectivity with the circuit underlying implicit modulation of emotion. In contrast, ELS was characterized by a compensatory pattern of intra-amygdaloid network causal connectivity that did not comport with customary anatomical and functional models of auditory threat and much more complex extra-amygdaloid connectivity. Behaviorally, modulation of threat was mediated through negative causal paths from vmPFC to BLA in non-ELS but through a unique intraamygdaloid BLA to CeA path in the ELS group indicative of disparate inhibitory mechanisms. These findings indicate that ELS disrupts modulation of emotion both at the nexus of threat detection within the amygdala as well as with other circuits throughout the brain.

\section{Limitations}

Limitations to the current study include the decision to employ conventional functional imaging methods and as such concerns regarding spatial resolution may be posed. However, prior investigations that have employed conventional acquisition methods have demonstrated functional and temporal differentiation between amygdala subregions (Morris et al, 2001; Etkin et al, 2009). Specifically, a number of prior investigations of localized amygdala subregion activity and rs-connectivity have employed $6 \mathrm{~mm}$ smoothing kernals (Etkin et al, 2009; Roy et al, 2009; Brown et al, 2014), whereas we employed a more conservative $4 \mathrm{~mm}$ kernel similar to Gamer et al (2010). Moreover, the current findings are consistent with animal models of chronic stress and amygdala subregion response (Rosenkranz et al, 2010). In addition we employed two additional measures to address this concern. First, the high-resolution structural image employed for localization of response was based on a template derived from the group average of the current sample and not a standard template, thus enhancing accuracy. Second, we compared response by subregion and observed disparate connectivity patterns. 
a

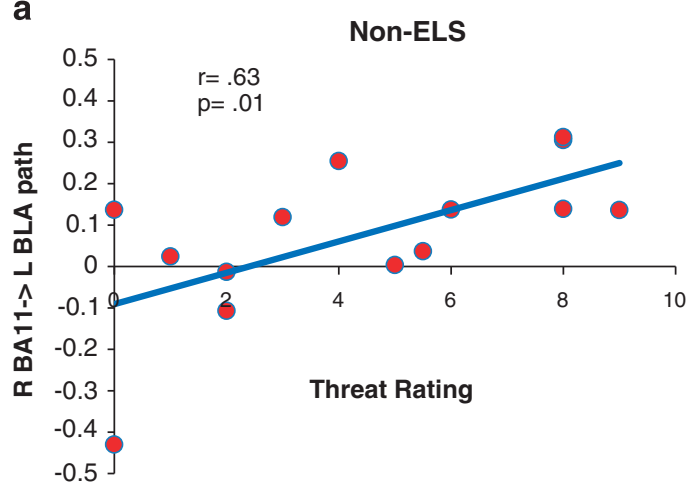

C

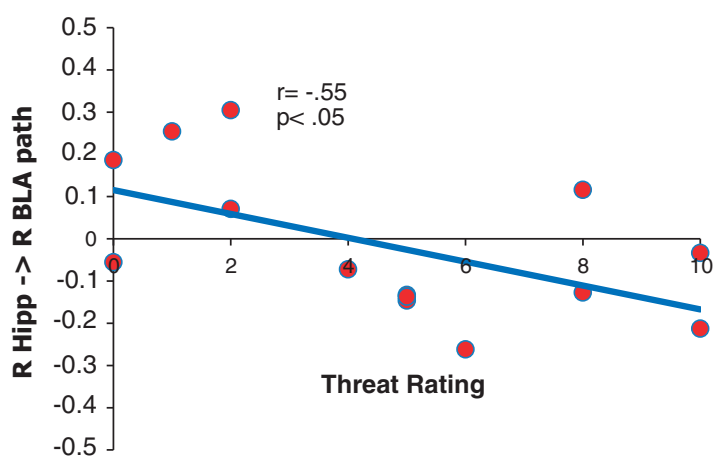

b

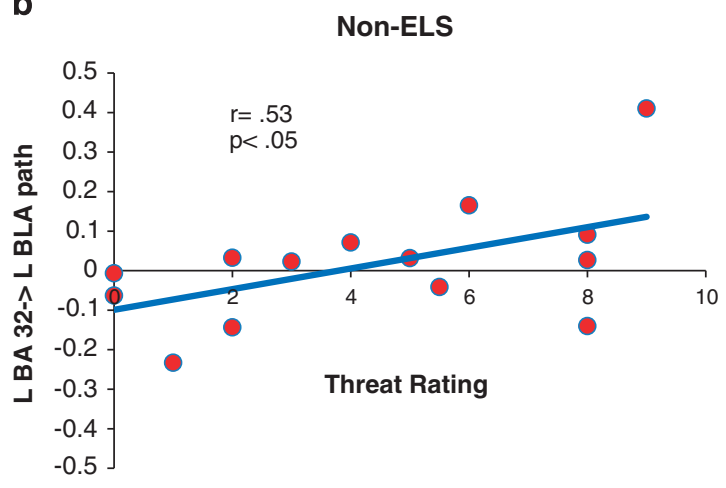

d

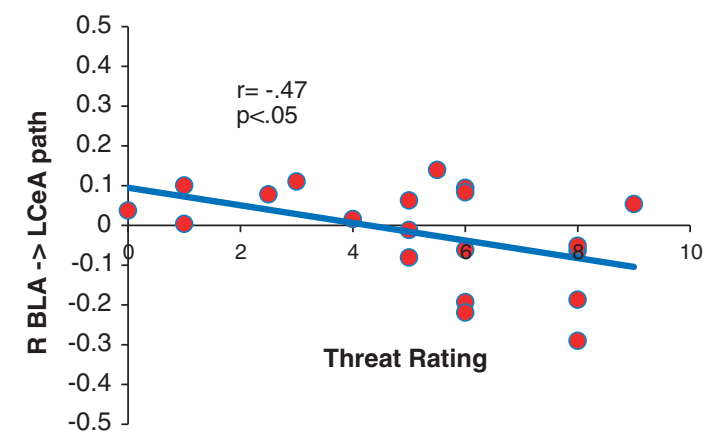

Figure 5 Granger Causality Paths and Implicit Modulation of Emotion. As a measure of implicit regulation of emotion, we performed correlation analysis between connectivity coefficients for intra-amygdaloid and extra-amygdaloid lateral/medial paths with individual threat ratings for the UCS trials. (a) A negative causal path from right BA I I (OFC) to left BLA was associated with less perceived threat in non-ELS, $p<0.0$ I (b) Similarly, a negative causal path from left BA32 to left BLA was associated with less perceived threat in the non-ELS group, $p<0.05$ (c) A positive causal path from right hippocampus to right BLA was associated with less perceived threat in the non-ELS group, $p<0.05$ and (d) A positive causal path from right BLA to left CeA was associated with less perceived threat within the ELS group, $p<0.05$. Abbreviations: BA, Brodmann's Area; BLA, basolateral; CeA, central nucleus; ELS, early life stress; OFC, orbitofrontal cortex; UCS, unconditioned stimulus.

Moreover, temporal resolution of fMRI is a limiting factor when employing Granger causality, which relies on lag-time. However, using a simple auditory-motor paradigm, Abler et al (2006) demonstrated that Granger causality can correctly estimate directional influences from the auditory cortex to the motor cortex (which is expected in this paradigm) even when fMRI data are acquired with a long TR of $2440 \mathrm{~ms}$. They argue that although a lack of significant Granger causality obtained from slowly sampled fMRI data does not imply a corresponding lack of directional influence at the neural level, significant Granger causality obtained from slowly sampled fMRI data is in fact likely to correctly reflect corresponding neural causality. The results presented in this study must be interpreted with this caveat in mind.

\section{FUNDING AND DISCLOSURE}

DCK's work has been funded by the NIH. The NIMH had no role in study design; in the collection, analysis and interpretation of data; in the writing of the report; or in the decision to submit the paper for publication. The remaining authors declare no conflict of interest.

\section{ACKNOWLEDGMENTS}

We would like to thank Baxter Rogers and the staff of the Vanderbilt University Institute of Imaging Science (VUIIS) for their assistance with imaging methods; Lauren Johnson, Joshua Shumen, Sara Robicheaux, and Samineh Hiebert for their assistance with data acquisition and general administrative assistance.

\section{REFERENCES}

Abler B, Roebroeck A, Goebel R, Schfnfeldt-Lecuona C, Hole G, Walter H (2006). Investigating directed influences between activated brain areas in a motor-response task using fMRI. Magn Reson Imaging 24: 181-185.

Amaral DG, Price JL, Pitkanen A, Carmichael ST (1992). Anatomical organization of the primate amygdaloid complex. In: Aggleton J (ed). The Amygdala: neurobiological Aspects of Emotion, Memory, and Mental Dysfunction. Wiley-Liss: New York, NY, USA, pp 1-66.

Amunts K, Kedo O, Kindler M, Pieperhoff P, Mohlberg H, Shah NJ et al (2005). Cytoarchitectonic mapping of the human amygdala, hippocampal region and entorhinal cortex: intersubject variability and probability maps. Anat Embryol (Berl) 210: 343-352. 
Bach D, Behrens T, Garrido L, Weiskopf N, Dolan R (2011). Deep and superficial amygdala nuclei projections revealed in vivo by probabilistic tractography. J Neurosci 31: 618-623.

Banks S, Eddy K, Angstadt M, Nathan P, Phan KL (2007). Amygdala-frontal connectivity during emotion regulation. Soc Cogn Affect Neurosci 2: 303-312.

Bernstein D, Fink LA (1998). Manual for the Childhood Trauma Questionnaire. The Psychological Corporation: New York, NY, USA.

Brown VM, LaBar KS, Haswell CC, Gold AL, McCarthy G, Morey RA (2014). Altered resting-state functional connectivity of basolateral and centromedial amygdala complexes in posttraumatic stress disorder. Neuropsychopharmacology 39: 351-359.

Dannlowski U, Stuhrmann A, Beutelmann V, Zwanzger P, Lenzen T, Grotegerd D et al (2012). Limbic scars: long-term consequences of childhood maltreatment revealed by functional and structural Magn Reson Imaging. Biol Psychiatry 71: 286-293.

Dannlowski U, Kugel H, Huber F, Stuhrmann A, Redlich R, Grotegerd D et al (2013). Childhood maltreatment is associated with an automatic negative emotion processing bias in the amygdala. Hum Brain Mapp 34: 2899-2909.

Davis M (2006). Neural systems involved in fear and anxiety measured with fear-potentiated startle. Am Psychol 61: 741-756.

Duvarci S, Pare D (2007). Glucocorticoids enhance the excitability of principal basolateral amygdala neurons. J Neurosci 27: 4482-4491.

Eickhoff SB, Stephan KE, Mohlberg H, Grefkes C, Fink GR, Amunts $\mathrm{K}$ et al (2005). A new SPM toolbox for combining probabilistic cytoarchitectonic maps and functional imaging data. Neuroimage 25: 1325-1335.

Etkin A, Prater K, Schatzberg A, Menon V, Greicius M (2009). Disrupted amygdalar subregion functional connectivity and evidence of a compensatory network in generalized anxiety disorder. Arch Gen Psychiatry 66: 1361-1372.

Fink LA, Bernstein D, Handelsman L, Foote J, Lovejoy M (1995). Initial reliability and validity of the childhood trauma interview: a new multidimensional measure of childhood interpersonal trauma. Am J Psychiatry 152: 1329-1335.

First MB, Spitzer RL, Gibbon M, Williams JBW (2002). Structured Clinical Interview for DSM-IV-TR Axis Disorders, Research Version, Non-Patient Edition (SCID-I/NP). Biometrics Research, New York State Psychiatric Institute: New York, NY, USA.

Freese J, Amaral D (2009). Neuroanatomy of the primate amygdala. In: Whalen P, Phelps E, editors. The human amygdala. New York, Guilford. 3-42.

Gamer M, Zurowski B, Buchel C (2010). Different amygdala subregions mediate valence-related and attentional effects of oxytocin in humans. Proc Natl Acad Sci USA 107: 9400-9405.

Ghashghaei HT, Barbas H (2002). Pathways for emotion: interactions of prefrontal and anterior temporal pathways in the amygdala of the rhesus monkey. Neuroscience 115: 1261-1279.

Ghashghaei HT, Hilgetag CC, Barbas H (2007). Sequence of information processing for emotions based on the anatomic dialogue between prefrontal cortex and amygdala. Neuroimage 34: 905-923.

Granger C (1969). Investigating causal relations by econometric models and cross-spectral methods. Econometrica 37: 424-438.

Grant MM, Cannistraci C, Hollon SD, Gore J, Shelton R (2011). Childhood trauma history differentiates amygdala response to sad faces within MDD. J Psychiatr Res 45: 886-895.

Grant MM, Hadley J, Hutcheson N, Shelton R, Sreenivasan K, Deshpande G (2014). Early life trauma and directional brain connectivity in major depression. Hum Brain Mapp 35: 4815-4826.

Green JG, McLaughlin KA, Berglund PA, Gruber MJ, Sampson NA, Zaslavsky AM et al (2010). Childhood adversities and adult psychiatric disorders in the national comorbidity survey replication I: associations with first onset of DSM-IV disorders. Arch Gen Psychiatry 67: 113-123.

Gross JJ, Thomspons RA (2007). Emotion regulation: conceptual foundations. In: Gross JJ (ed). Handbook of Emotion Regulation. Guilford Press: New York, NY, USA, pp 3-24.

Havlicek M, Friston K, Jan J, Brazdil M, Calhoun V (2011). Dynamic modeling of neuronal responses in fMRI using cubature Kalman filtering. Neuroimage 56: 2109-2128.

Kalisch R (2009). The functional neuroanatomy of reappraisal: time matters. Neurosci Biobehav Rev 33: 1215-1226.

LaBar KS, LeDoux JE (2011). Coping with danger: the neural basis of defensive behavior and fearful feelings. Compr Physiol 17: 139-154.

LeDoux JE (2007). The amygdala. Curr Biol 17: R868-R874.

Marusak HA, Martin KR, Etkin A, Thomason ME, Vaitl D, Stark R (2014). Childhood trauma exposure disrupts the automatic regulation of emotional processing. Neuropsychopharmacology (in press).

Merz CJ, Tabbert K, Schweckendiek J, Klucken T, Vaitl D, Stark R et al (2010). Investigating the impact of sex and cortisol on implicit fear conditioning with fMRI. Psychoneuroendocrinology 35: 33-46.

Merz CJ, Wolf OT, Schweckendiek J, Klucken T, Vaitl D, Stark R (2013). Stress differentially affects fear conditioning in men and women. Psychoneuroendocrinology 38: 2529-2541.

Meyer-Lindenberg A, Olsen R, Kohn P, Brown T, Egan MF, Weinberger DR et al (2005). Regionally specific disturbance of dorsolateral prefrontal-hippocampal functional connectivity in schizophrenia. Arch Gen Psychiatry 62: 379-386.

Morris JS, Buchel C, Dolan RJ (2001). Parallel neural responses in amygdala subregions and sensory cortex during implicit fear conditioning. Neuroimage 13: 1044-1052.

Philip NS, Sweet LH, Tyrka AR, Price LH, Bloom RF, Carpenter LL (2013). Decreased default network connectivity is associated with early life stress in medication-free healthy adults. Eur Neuropsychopharmacol 23: 24-32.

Phillips ML, Ladouceur CD, Drevets WC (2008). A neural model of voluntary and automatic emotion regulation: implications for understanding the pathophysiology and neurodevelopment of bipolar disorder. Mol Psychiatry 13: 829, 833-857.

Price JL, Drevets WC (2010). Neurocircuitry of mood disorders. Neuropsychopharmacology 35: 192-216.

Quirk G, Likhtik E, Pelletier J, Pare D (2003). Stimulation of medial prefrontal cortex decreases the responsiveness of central amygdala output neurons. J Neurosci 23: 8800-8807.

Radley JJ, Sisti HM, Hao J, Rocher AB, McCall T, Hof PR et al (2004). Chronic behavioral stress induces apical dendritic reorganization in pyramidal neurons of the medial prefrontal cortex. Neuroscience 125: 1-6.

Rodrigues SM, LeDoux JE, Sapolsky RM (2009). The influence of stress hormones on fear circuitry. Annu Rev Neurosci 32: 289-313.

Rosenkranz JA, Venheim ER, Padival M (2010). Chronic stress causes amygdala hyperexcitability in rodents. Biol Psychiatry 67: $1128-1136$

Roy AK, Shehzad Z, Margulies DS, Kelly AM, Uddin LQ, Gotimer K et al (2009). Functional connectivity of the human amygdala using resting state fMRI. Neuroimage 45: 614-626.

Schiller D, Levy I, Niv Y, LeDoux JE, Phelps EA (2008). From fear to safety and back: reversal of fear in the human brain. J Neurosci $\mathbf{2 8}$ 11517-11525.

Shin LL, Liberzon I (2010). The neurocircuitry of fear, stress and anxiety disorders. Neuropsychopharmacology 35: 169-191.

Shin LM, Wright CI, Cannistraro PA, Wedig MM, McMullin K, Martis B et al (2005). A functional Magn Reson Imaging study of amygdala and medial prefrontal cortex responses to overtly presented fearful faces in posttraumatic stress disorder. Arch Gen Psychiatry 62: 273-281. 
Silvers JA, Wager TD, Weber J, Ochsner KN (2014). The neural bases of uninstructed negative emotion modulation. Soc Cogn Affect Neurosci 10: 10-18.

Stein JL, Wiedholz LM, Bassett DS, Weinberger DR, Zink CF, Mattay VS et al (2007). A validated network of effective amygdala connectivity. Neuroimage 36: 736-745.

Treadway MT, Grant MM, Ding Z, Hollon SD, Gore JC, Shelton RC (2009). Early adverse events, HPA activity and rostral anterior cingulate volume in MDD. PloS One 4: e4887.

Vyas A, Bernal S, Chattarji S (2003). Effects of chronic stress on dendritic arborization in the central and extended amygdala. Brain Res 965: 290-294.
Wheelock MD, Sreenivasan KR, Wood KH, Ver Hoef LW, Desphande G, Knight DC (2014). Threat-related learning relies on distinct dorsal prefrontal cortex network connectivity. Neuroimage 102(Pt 2): 904-912.

Williams LM, Kemp AH, Felmingham K, Barton M, Olivieri G, Peduto A et al (2006). Trauma modulates amygdala and medial prefrontal responses to consciously attended fear. Neuroimage 29: 347-357.

Wood KH, Ver Hoef LW, Knight DC (2012). Neural mechanisms underlying the conditioned diminution of the unconditioned fear response. Neuroimage 60: 787-799.

Zald DH (2003). The human amygdala and the emotional evaluation of sensory stimuli. Brain Res Brain Res Rev 41: 88-123.

Supplementary Information accompanies the paper on the Neuropsychopharmacology website (http://www.nature.com/npp) 